Universidad de Lima

Facultad de Ingeniería y Arquitectura

Carrera de Ingeniería de Sistemas

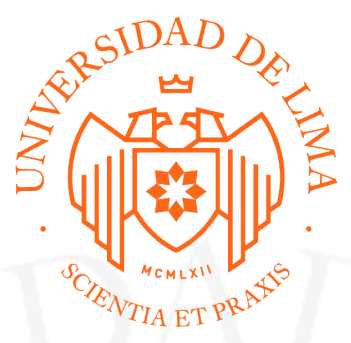

Análisis de Patrones en Señales Musculares de Extremidad Superior Mediante Técnicas de Machine Learning

Trabajo de investigación para optar el grado académico de bachiller en Ingeniería de Sistemas

Diana del Milagro Ayala Galvan

Código 20152736

\author{
Asesores \\ Juan Manuel Gutiérrez Cárdenas \\ Carlos Andrés Mugruza Vasallo
}

Lima - Perú

Setiembre de 2019 


\title{
Análisis de Patrones en Señales Musculares de Extremidad Superior Mediante Téenicas de Machine Learning
}

\author{
Autor(es) \\ Ayala Galvan, Diana del Milagro \\ 20152736@aloe.ulima.edu.pe \\ Universidad de Lima
}

\section{Resumen:}

Con la finalidad de encontrar las mejores técnicas para el análisis de señales electromiográficas (EMG) a través de medios computacionales para su uso en el control de prótesis, se realizaron dos clasificadores de señales. En este artículo se detallará la metodología empleada tanto en la obtención de las señales, como en la implementación del clasificador.

Metodología: Recolección de señales EMG mediante el sensor analógico Myoware Muscle Sensor y la placa Arduino Mega 2560. Implementación de clasificadores mediante TensorFlow ${ }^{\mathrm{TM}}$ y Scikit Learn para redes neuronales convolucionales (CNN) y perceptrón multicapa (MLP), respectivamente.

Resultados: Final test accuracy de 60\% y 33.33\% para CNN y MLP, de manera correspondiente.

Conclusiones y futuros trabajos: Se requiere un análisis más profundo para este tipo de señales, con la finalidad de incrementar la precisión de los modelos realizados.

\section{Palabras Clave:}

Prótesis, Extremidad superior, Machine Learning, Análisis de Señales.

\begin{abstract}
:
In order to find the best techniques to analyze EMG signals with computational processes for its use in prosthetic control, two signal classifiers were implemented. This article will detail the methodology used both in obtaining the signals, and in the implementation of the classifier.
\end{abstract}

Methodology: EMG signal collection using the Myoware Muscle Sensor analogue sensor and the Arduino Mega 2560 board. Implementation of classifiers using TensorFlow TM and Scikit Learn for convolutional neural networks (CNN) and multilayer perceptron (MLP), respectively.

Results: Final test accuracy of $60 \%$ and $33.33 \%$ for CNN and MLP, correspondingly.

Conclusions and future work: A deeper analysis is required for this type of signals, in order to increase the accuracy of the models performed.

\section{Keywords:}

Prosthetics, Upper limb, Machine Learning, Signal Analysis.

\section{INTRODUCCIÓN}

Una fracción considerable de los peruanos presentan algún tipo de amputación; mas no muchos tienen acceso a prótesis de calidad debido los costos elevados que estas suponen, lo cual limita las posibilidades del paciente a desarrollarse de manera cotidiana (Baldwin \& Johnson, 2014). Uno de los beneficios brindados por las prótesis consiste en poder superar la depresión generada por el cambio en su imagen corporal y funcionalidades, que, a su vez, elimina la necesidad de un tercero y nivela las oportunidades laborales a las que el paciente puede acceder (Baldwin \& Johnson, 2014).

Asimismo, el poseer una prótesis elaborada con materiales adecuados se reduce la probabilidad de enfermedades que afectan al muñón del paciente, al ser una zona delicada y expuesta. (Highsmith, Highsmisth, \& Kahle, 2011). 
Favorece, también, la recuperación del trastorno de la "extremidad fantasma”, el cual es padecido por el 79, 9\% de los pacientes con alguna amputación y consiste en sentir dolor en la extremidad que ya no se posee (Dilck, y otros, 2014), (Ephrain, Wegener, Mackenzie, Dillingham, \& Pezzin, 2005).

Por otro lado, son numerosas las prótesis en el mercado que poseen un sistema de recolección de señales de calidad, pero no son accesibles en cuanto al entendimiento del paciente para usarlas de manera correcta, por lo que varios usuarios prefieren emplear prótesis mecánicas. (Hunter, Heger, \& Millstein, 1986)

La presente investigación tiene como objetivo realizar un análisis adecuado de señales obtenidas desde los músculos de la extremidad superior intacta de diferentes sujetos de prueba. Esto se realiza con la finalidad de, en un futuro, lograr encontrar la técnica adecuada para el procesamiento de las señales musculares que permitan implementar un sistema de clasificación de movimientos para una prótesis.

\section{ANTECEDENTES}

En el Perú, aproximadamente el 3.08 \% de la población padece de algún tipo de discapacidad y presenta una dificultad para moverse, caminar y/o usar brazos y piernas; sin embargo, solo el 0.3\% tiene acceso a una prótesis (INEI, 2012).

Según un estudio realizado en el Instituto Nacional de Rehabilitación (INR), el 57.1\% y 25.4\% de los pacientes con alguna amputación pertenecen a un nivel socioeconómico pobre no extremo y pobre extremo respectivamente (Farro, Tapia, Bautista, Montalvo, \& Iriarte, 2012).

Lo previamente mencionado dificulta el acceso a una prótesis de calidad, ya que estas suelen tener un precio que sobrepasa las decenas de miles de dólares.

Por otro lado, las prótesis de alto rendimiento requieren de recursos tecnológicos que son escasos y limitados en el país (Dorador González, 2004). Esto sumado a que se cuenta con escasa oferta de mano de obra de especialistas interesados en el área de robótica (Ñique, 2011).

Asimismo, la dificultad de las prótesis radica en su finalidad, ya que buscan suplir las funcionalidades de un brazo o pierna natural, los cuales requieren de altos grados de precisión, por lo que es imprescindible realizar estudios detallados y exhaustivos para ser replicados por un sistema robótico de manera eficiente y al menos costo posible (Ñique, 2011).

Otra razón por la que son necesarios estos estudios es el requerimiento de personalización de las mismas, debido a que cada paciente presenta diferentes tipos de amputación con tamaños heterogéneos de miembros residuales, así como requieren de distintos niveles de funcionalidad, por lo que cada una de las prótesis suele manufacturarse solo a pedido.

\section{ESTADO DEL ARTE}

\subsection{Estándares de control empleados actualmente en el control de las prótesis}

A lo largo de los años, diversos tipos de prótesis controladas a través de diferentes mecanismos se han desarrollado en diversos estudios. Por lo general, son cuatro los tipos de control más comunes:

Prótesis controlada a través de Interfaces Cerebro - Máquina (Brain Machine Interface - BMI): Tanto el cerebro humano como animal son capaces de emitir señales cerebrales. Los sistemas BMI se encargan de transmitir dichas señales hacia determinados dispositivos externos, traduciéndolos en señales de control que genera una salida. Estas señales se obtienen colocando electrodos en la parte inferior del cráneo, ya sea debajo o sobre la duramadre. En comparación con otros tipos de señales, estas presentan alta resolución espacial y fidelidad de la señal, resistencia al ruido y robustez sustancial durante largos períodos de grabación, lo que genera una mayor precisión. (Schalk \& Leuthardt, 2011)

Prótesis controlada a través de señales electromiográficas de superficie (sEMG): Consiste en la detección de potenciales de activación de los músculos del muñón del paciente (Orabona, Castellini, Caputo, Fiorilla, \& Sandini, 2009). Actualmente, son pocos los casos en los que este tipo de control se emplean para un agarre preciso, usando por lo general movimientos predefinidos. El caso más avanzado es el desarrollado por la empresa Touch Bionics en la 
prótesis comercial i-Limb, donde se le permite al usuario elegir entre un conjunto predefinido de formas de agarre (Cipriani, Zaccone, Micera, \& Carrozza, 2008).

Prótesis controlada a través de señales mioeléctricas: Mediante el uso de sensores electrónicos, se detecta actividad de los músculos y nervios. Esta actividad se traduce en información empleada como mecanismo de control para este tipo de prótesis. Mediante este tipo de control, el usuario puede llegar a ser capaz de controlar la fuerza y la velocidad de los movimientos, así como del agarre de la extremidad variando la intensidad muscular (Ashton Dalley, Varol, \& Goldfarb, 2012).

Prótesis controlada a través del habla: Consiste en operar la prótesis mediante determinados fonemas expresados por el usuario y recogidos por un software de reconocimiento de voz. Si bien este tipo de control facilita las tareas a aquellas personas con escaso control muscular, solo se limita a comandos discretos y falla en tareas direccionales que requieren de un control bastante preciso. (House, Malkin, \& Bilmes, 2009)

\subsection{Métodos y algoritmos empleados actualmente en el control de prótesis con alta precisión y respuestas en tiempo real}

Con la finalidad de generar una mayor precisión, en la implementación de prótesis de extremidades se hace uso de diferentes tipos de algoritmos en el procesamiento de las señales de control.

Field Programmable Gate Array (FPGA): Los dispositivos FPGA son aquellos cuya estructura lógica final puede ser configurada o programada por el usuario final sin la necesidad de una instalación de circuitos integrados a nivel de hardware (Brown, Francis, Rose, \& Vranesic, 1992).

Máquina de Estado Finito (Finite State Machine - FSM): Es un modelo matemático computacional que, en determinado momento, se puede encontrar en uno de un número finito de estados, y puede variar entre estos como respuesta a un determinado valor de entrada. En el caso de prótesis, estos sistemas se emplean para definir una arquitectura basada en el periodo de entrenamiento (estado 1) y el periodo de prueba (estado 2), donde se realizan funciones completamente diferentes (Huang, et al., 2010).

Redes Neuronales Artificiales: Sistemas computacionales basados en las conexiones neuronales biológicas del cerebro, con la finalidad de que este sistema sea capaz de aprender con el tiempo. Esto quiere decir que el sistema será capaz de mejorar de manera progresiva el desempeño de sus tareas y asignaciones mediante las repeticiones de estas. Estos sistemas son empleados para la detección de patrones en las señales, relacionándolos con los movimientos pretendidos por el usuario (Hiraiwa, Shimohara, \& Tokunaga, 1989).

Support vector machines (SVM): Las máquinas de soporte vectorial o SVM son métodos de aprendizaje automático capaces de determinar la mejor función para la resolución de un problema de regresión o clasificación, extraído de un espacio funcional inducido por la elección de una función binaria entre puntos en el espacio de entrada. Por lo general, esta técnica es empleada como método de clasificación de los vectores generados por la data obtenida por EMG (Castellini \& Van der Smagt, 2009).

\subsection{Métodos y materiales empleados actualmente en la elaboración del socket y demás partes físicas de la prótesis}

Tradicionalmente, el socket de las prótesis se fabrica a través de un procedimiento manual. Por este motivo, la forma final depende en gran medida de las habilidades y experiencia del especialista. En este procedimiento no se toman en cuenta varios aspectos relativos a la dureza del material, lo que suele generar fatiga y dolencia en los pacientes a lo largo de la zona de carga y descarga (Zhang, Huang, \& Yang, 2012). Por esta razón, las tendencias actuales buscan popularizar el desarrollo de sockets para prótesis a través de impresoras $3 \mathrm{D}$, lo que permitiría tanto una automatización del proceso como una mayor precisión en el desarrollo de estos (Zhang, Huang, \& Yang, 2012).

\section{METODOLOGÍA}

\subsection{Propuesta de Solución}


Con la finalidad de mejorar la situación detallada en la sección anterior, se propone la implementación de un sistema de procesamiento de señales musculares, el cual permitirá realizar el análisis de patrones de dichas señales a través de técnicas de Machine Learning. Esto se logrará a través de dos fases, detalladas a continuación:

Fase I: Recolección de señales mioeléctricas: Recolección de información proveniente de los sensores mioeléctricos.

Fase II: Procesamiento y análisis de las señales: Selección del movimiento a través de una red neuronal.

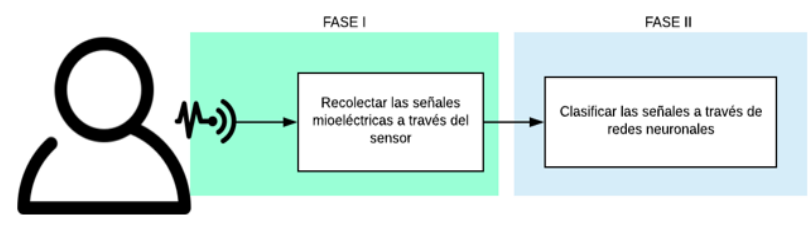

Fig. 1. Flujograma del sistema propuesto. Fuente: Elaboración propia.

\subsection{Prueba de Concepto}

Con la finalidad de demostrar que es posible clasificar señales EMG haciendo uso de redes neuronales, se realizará la implementación de dos clasificadores de señales EMG aplicando dos tipos de redes neuronales artificiales: Redes Neuronales Convolucionales y Perceptrón Multicapa. Para ello, se requerirá realizar la toma de señales respectiva, detallada a continuación. Cabe destacar que todos los procedimientos realizados y descritos a continuación, fueron ejecutados en una MacBook Pro 2017 TouchBar, con 8 GB de memoria RAM, y haciendo uso del sistema operativo OSX High Sierra.

\section{Obtención de señales EMG}

Para la presente investigación, en primera instancia, se realizó la toma de señales electromiográficas a doce sujetos mediante el uso del sensor analógico Myoware Muscle Sensor (Fig 2) y la placa programable de hardware libre Arduino Mega 2560 (Fig. 3). El sensor fue colocado en el bíceps, tal como se muestra en la figura 4. A los usuarios se les pidió la realización de tres movimientos determinados, detallados a continuación:

- Movimiento A: Abrir la mano con la mayor fuerza posible.

- Movimiento B: Cerrar la mano con los músculos del brazo, mas no del antebrazo.

- Movimiento C: Cerrar la mano tanto con los músculos del brazo como del antebrazo.

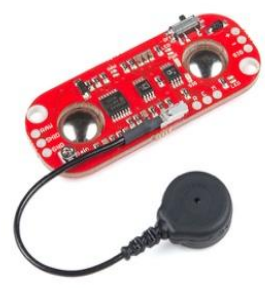

Fig. 2. Myoware Muscle Sensor. Recuperado de: https://cdn.sparkfun.com//assets/parts/1/1/2/3/3/13723-MyoWare_Muscle_Sensor-02.jpg

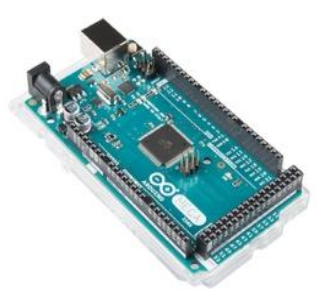




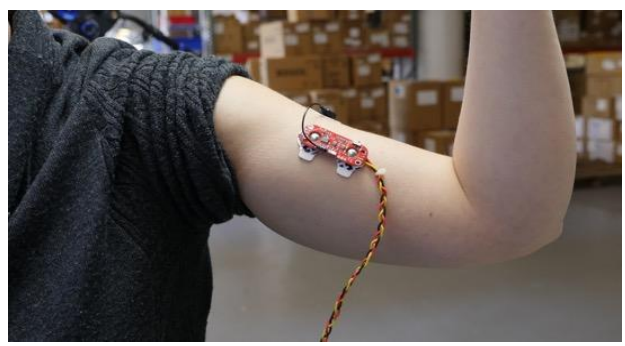

Fig. 4. Colocación del sensor en el antebrazo del sujeto. Recuperado de: https://cdnlearn.adafruit.com/assets/assets/000/030/042/large1024/biometric_MyoWare bicep.jpg?1453783907

En cuanto el tiempo a emplear para la realización del movimiento, se les pidió a los usuarios que en cada iteración demoren lo que consideren pertinente, dado que, en un ámbito real, es así como se producen los movimientos. La frecuencia usada para este análisis fue de $2 \mathrm{~Hz}$.

A través de dichas medidas, se pudo apreciar una existencia de saturación (Fig. 5) en ciertos sujetos. Ello evidenció un problema en el muestreo realizado. Además, al realizar el espectrograma de dichas medidas con el análisis sobre un solo bit, las características de estos movimientos no pudieron ser apreciadas adecuadamente (Fig. 6).

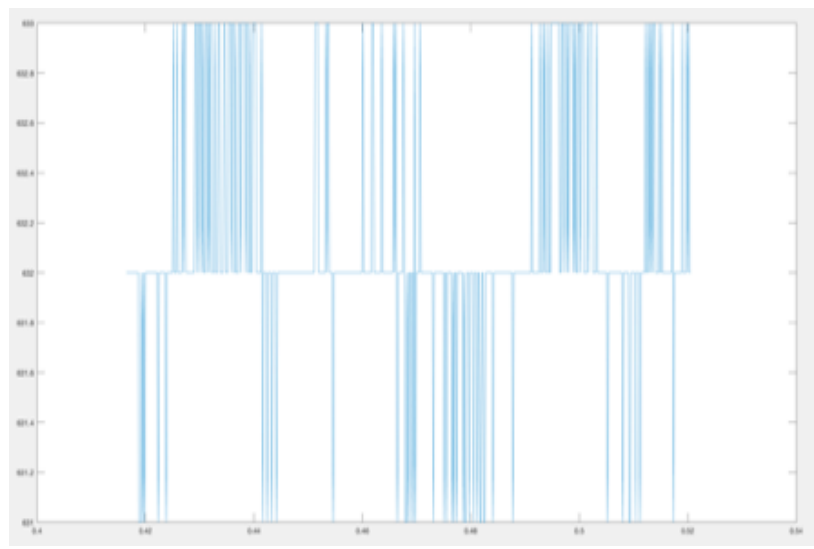

Fig. 5. Señal saturada. Fuente: Elaboración propia a través de MatLab

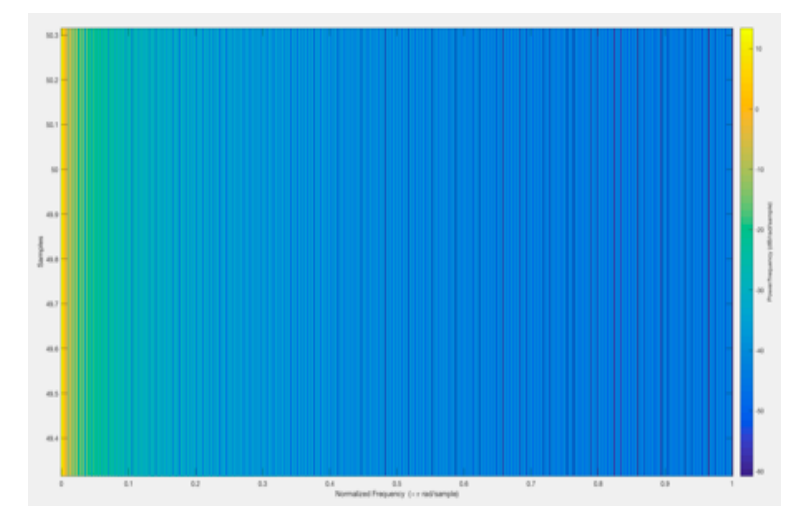

Fig. 6. Espectrograma de 1 bit. Fuente: Elaboración propia a través de MatLab

Posteriormente, las señales obtenidas fueron filtradas a través de la librería " $<$ FiltroKalman.h $>$ " de Arduino, de las cuales se generó el espectrograma y se procedió a realizar la clasificación de las señales mediante redes convolucionales.

Se obtuvo como resultado un train accuracy, validation accuracy y final test accuracy de 100\%, así como un valor de cross entropy de 0.000626 . Esto evidencia problemas de muestro, baja cantidad de data, así como overfitting del modelo. Los indicadores mencionados serán explicados más adelante. 


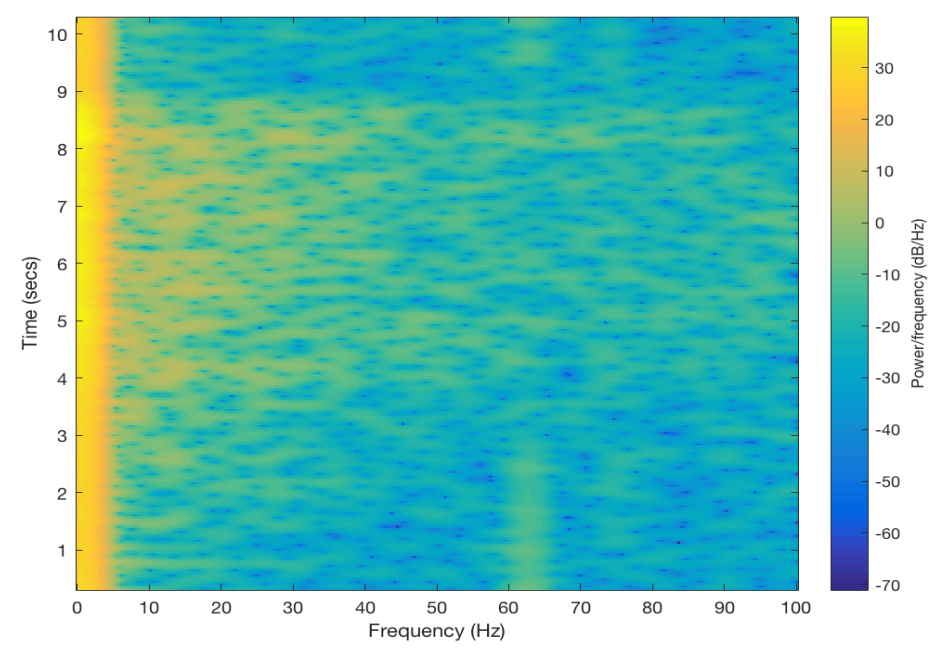

Fig. 7. Espectrograma de 512 bits. Fuente: Elaboración propia a través de MatLab

Fue por este motivo que se procedió a volver a realizar el muestreo de señales, haciendo uso de una frecuencia de 200 $\mathrm{Hz}$ con la finalidad de obtener un muestreo más preciso. No obstante, y dado que cada persona mostró diferentes características propias de sí mismas diferenciando así sus movimientos de los de las otras personas, el muestreo realizado en segunda instancia fue en función de una sola persona, solicitándole realizar los movimientos previamente descritos para veinte muestras de cada uno de los mismos.

Las señales obtenidas fueron graficadas como espectrogramas en MatLab, haciendo uso de 512 bits (Fig. 7) para el análisis. Teniendo como base los archivos .txt de las señales obtenidas, así como dichos espectrogramas en formato .jpg, se realizó lo detallado en la siguiente subsección.

\section{Clasificador de Espectrogramas de Señales EMG con Redes Neuronales Convolucionales}

A través de la librería TensorFlow ${ }^{\mathrm{TM}}$ y su red neuronal convolucional pre entrenada en el reconocimiento de imágenes, se implementó un clasificador de señales EMG. Para ello, se recolectaron los espectrogramas de las señales EMG en un mismo directorio, etiquetándolos según el movimiento correspondiente. Posteriormente, se ejecutaron los comandos para iniciar tensorboard en el terminal de la computadora, asignándoles el directorio creado con dicha finalidad como directorio de banco de imágenes de entrenamiento. El entrenamiento fue realizado mediante 4000 iteraciones, seleccionando de manera aleatoria cinco espectrogramas de la data de entrenamiento.

\section{Clasificador de Señales EMG con Perceptrón Multicapa}

Haciendo uso de la librería Scikit Learn de Python, se implementó un clasificador de señales EMG a partir de la red neuronal de tipo perceptrón multicapa. Los datos recolectados en formato ttxt fueron leídos por una función implementada por nosotros en Python. Este programa se realizó con la finalidad de convertir los archivos mencionados en arreglos de valores obtenidos. Debido a la variación en el tiempo de cada movimiento, los datos obtenidos no fueron de la misma longitud.

Por este motivo, y ya que cada valor obtenido se hará uso como feature para la red, existía una cantidad diferente de estos para cada movimiento muestreado. Como solución parcial a este problema, se procedió a rellenar aquellos arreglos con menor cantidad de características con el valor cero, de tal manera que todas las muestras tuviesen la misma cantidad de datos. El algoritmo realizó tan solo trece iteraciones durante la fase de entrenamiento debido a una falta de mejora en la función de pérdida.

Con este banco de datos, y sus respectivas etiquetas, se realizó el entrenamiento de la red, con la finalidad de que esta sea capaz de diferenciar entre los diferentes tipos de movimientos administrados. 


\subsection{Fast Fourier Transform (FFT) y filtro pasabanda}

Se aplicó FFT en las señales, con la finalidad de reducir la cantidad de datos obtenida, para así realizar un análisis más preciso en función de la frecuencia. No obstante, los resultados obtenidos mostraron que la frecuencia de esta señal se encuentra en un intervalo negativo. Esto demuestra la alta complejidad del análisis de Fourier, el cuál descompone la señal de manera exponencial compleja. (Cerna \& Harvey, 2000) Esto quiere decir que son espirales girando alrededor de un plano complejo. Debido a esto, no se pudo realizar el filtro pasabanda, el cual solo se encuentra implementado para frecuencias positivas en Matlab. Se requiere una investigación más profunda sobre la naturaleza de las señales musculares para hacer uso de este tipo de técnicas avanzadas.

\section{RESULTADOS}

\subsection{Clasificador de Espectrogramas de Señales EMG con Redes Neuronales Convolucionales}

TensorFlow $^{\mathrm{TM}}$ hace uso de cuatro principales indicadores para la red neuronal empleada en este experimento (TensorFlow, 2018), explicadas a continuación.

Train accuracy: Hace referencia al porcentaje de imágenes etiquetadas satisfactoriamente durante el entrenamiento realizado. (TensorFlow, 2018)

Validation accuracy: Hace referencia al porcentaje de imágenes seleccionadas aleatoriamente como validación del entrenamiento y etiquetadas satisfactoriamente durante la iteración actual. (TensorFlow, 2018)

Cross entropy: Hace referencia a la función de pérdida, la cual demuestra qué tanto está progresando el modelo. (TensorFlow, 2018)

Final test accuracy: Este número se basa en el porcentaje de las imágenes en el banco de datos de pruebas que reciben la etiqueta correcta después de que el modelo ha finalizado el entrenamiento. (TensorFlow, 2018)

Los resultados obtenidos durante este experimento fueron los siguientes:

Train accuracy (iteración 4000): 100\%

Cross entropy (iteración 4000): 0.001616

Validation accuracy (iteración 4000): 100\%

Final test accuracy: $60 \%(\mathrm{~N}=5)$

\subsection{Clasificador de Señales EMG con Perceptrón Multicapa}

La librería empleada solo pudo mostrar dos indicadores tras el entrenamiento realizado:

Función de pérdida o cross-entropy (Iteración 13): 1.10049726

Training set score: 0.333333

\section{DISCUSIÓN}

Como se puede apreciar en los resultados previamente mostrados, la precisión final tras el entrenamiento de ambos modelos no resulta alentadora.

En el caso del clasificador basado en redes convolucionales, claramente se observa un overfitting tras el entrenamiento. Esto se produce debido a que la cantidad de data, al parecer, no es la adecuada. Según TensorFlow ${ }^{\mathrm{TM}}$, la cantidad mínima de data que se le debe insertar a esta red para que el código logre funcionar es de veinte muestras por categoría. No obstante, afirman que entre mayor sea la cantidad de datos, mayor será la precisión. Para esta investigación se hizo uso de dicha cantidad, lo que parece ser el motivo de los resultados. Además, el dato de Final test accuracy refleja lo anterior. Debido a la existencia de este overfitting durante el entrenamiento, al intentar con data seleccionada como data de prueba, los resultados finales obtenidos no coinciden con los obtenidos durante dicho entrenamiento. 
Por otro lado, en el caso del perceptrón multicapa, los valores muestran que la data proporcionada al modelo no fue la correcta. Como se puede apreciar, la función de pérdida finalizó con un valor bastante alto, y solo realizó trece iteraciones debido a que esta no mejoró. Esto es debido a la pérdida de información que se dio al rellenar con valores cero a aquellos datos que no alcanzaron la longitud del máximo. Dado que muchos movimientos fueron realizados en dos o tres segundos, y algunos llegaron a cinco, el modelo no pudo determinar correctamente los tipos de movimiento, ya que muchos features fueron opacados por la cantidad de ceros empleadas en el arreglo.

\section{CONCLUSIONES}

Los modelos empleados para el presente análisis no lograron satisfacer las expectativas. Esto no es debido a que estos no sean adecuados, sino que la data proporcionada no brindaba la suficiente información para ambos casos. Cabe resaltar que, de tener una mayor cantidad de data, y analizarla de tal forma que la información no se vea opacada por data basura, los resultados a obtener, probablemente, serían mucho mejores. No obstante, con la presente investigación se ha podido llegar a la conclusión de que las señales EMG son bastante complejas, y requieren de un análisis y descomposición más profundas para poder realizar una clasificación precisa.

\section{TRABAJOS FUTUROS}

A fin de colaborar en la problemática detallada previamente, se propone realizar la clasificación nuevamente tras el análisis de las señales mediante Fast Fourier Transform, para, así, pasar la data obtenida al dominio de la frecuencia. Además, se deberán filtrar los datos obtenidos a través de un filtro pasabanda de manera adecuada, el cuál eliminará la data no necesaria. Se requiere la calibración del sensor empleado mediante un osciloscopio, tomando en cuenta la resistencia propia de cada persona.

\section{AGRADECIMIENTOS}

Mi más profundo agradecimiento a los profesores Carlos Mugruza y Juan Gutiérrez por su constante asesoría y preocupación para el logro de esta investigación.

\section{REFERENCIAS}

Ashton Dalley, S., Varol, H. A., \& Goldfarb, M. (2012). A Method for the Control of Multigrasp Myoelectric Prosthetic Hands. IEEE Transactions on Neural Systems and Rehabilitation Engineering, 58-67.

Atherton BSc, R. J. (2002). The role of Psychology in health and disability. In R. J. Atherton BSc, Psychological adjustment to lower limb amputation. Leicester: ProQuest.

Atzori, M., Gijsberts, A., Muller, H., \& Caputo, B. (2014). Classification of hand movements in amputated subjects by sEMG and accelerometers. 36th Annual International Conference of the IEEE Engineering in Medicine and Biology Society. https://doi.o.

Ayuyao Castillo, E., \& Ahmadinia, A. (2017). Distributed Deep Convolutional Neural Network For Smart Camera Image Recognition. ICDSC 2017.

Baldwin, M., \& Johnson, W. G. (2014). Labor Market Discrimination Against Men with Disabilities in the Year of the ADA. Southern Economic Journal.

Bengio, Y., \& Lecun, Y. (1998). Convolutional Networks for Images, Speech, and Time-Series. The handbook of brain theory and neural networks, 255-258.

Bhaskara Rao, P., Vara Prasad, D., \& Pavan Kumar, C. (2013). Feature Extraction Using Zernike Moments. International Journal of Latest Trends in Engineering and Technology, 228-234. 
Bornato, P., de Alessio, T., \& Knaflitz, M. (1998). A statistical method for the measurement of the muscle activation intervals from surface myoelectric signal gait. IEEE Trans Biomed Eng, 287-299.

Bouwsema, H., van der Sluis, C. K., \& Bongers, R. M. (2010). Learning to Control Opening and Closing a Myoelectric Hand. Archives of Physical Medicine and Rehabilitation, 1442-1446.

Breakey, J. W. (1997). Body Image: The Lower-Limb Amputee. Journal of prosthetics and orthotics.

Brown, S. D., Francis, R. J., Rose, J., \& Vranesic, Z. G. (1992). Field-Programmable Gate Arrays. Springer.

Carmena, J. M. (2013). Advances in Neuroprosthetic Learning and Control. PLOS ONe, 11(5).

Castellini, C., \& Van der Smagt, P. (2009). Surface EMG in Advanced Hand Prosthetics. Byological Cybernetics.

Castillo, A., Vázquez, J., Ortegón, J., \& Rodríguez, C. (2008). Prácticas de laboratorio para estudiantes de ingeniería con FPGA. IEEE LATIN AMERICA TRANSACTIONS, 130-136.

Chun-jing, T., Li, L., \& Xiao-yu, Z. (2010). Clinical study of phantom limb pain by fitting prosthesis at early stage after amputation. Conference: Proceedings of the 4th International Convention on Rehabilitation Engineering \& Assistive Technology.

Cipriani, C., Zaccone, F., Micera, S., \& Carrozza, M. (2008). On the Shared Control of an EMG-Controlled Prosthetic Hand: Analysis of User-Prosthesis Interaction. IEEE Transactions on Robotics, 170-184.

Ciresan, D. C., Meier, U., Masci, J., Gambardella, L. M., \& Schmidhuber, J. (2011). Flexible, High Performance Convolutional Neural Networks for Image Classification. Proceedings of the Twenty-Second International Joint Conference on Artificial Intelligence, 1237-1242.

Comotti, C., Regazzoni, D., Rizzi, C., \& Vitali, A. (2015). Multi-material design and 3D printing method of lower limb prosthetic sockets. Proceedings of the 3rd 2015 Workshop on ICTs for Improving Patients Rehabilitation Research Techniques, 42-45.

Dilck, D., Safaz, I., Adigüzel, E., Uran, A., Sarsoy, G., Goktepe, A. S., \& Tan Arif, K. (2014). The relationship between prosthesis use, phantom pain and psychiatric symptoms in male traumatic limb amputees. Comprehensive Psychiary.

Dorador González, J. (2004). Robótica y Prótesis Inteligentes. Revista Digital Universitaria.

Ephrain, P. L., Wegener, S. T., Mackenzie, E. J., Dillingham, T. R., \& Pezzin, L. E. (2005). Phantom Pain, Residual Limb Pain, and Back Pain in. Arch Phys Med Rehabil.

Fang, J., Agarwal, G. C., \& Shahani, B. T. (1997). Decomposition of EMG signals by wavelet spectrum matching. Procedures of the 19th Annual International Conference of the IEEE Engineering in Medicine and Biology Society 1997, 1253-1256.

Farro, L., Tapia, R., Bautista, L., Montalvo, R., \& Iriarte, H. (2012). Características clínicas y demográficas del paciente amputado. Revista Médica Herediana, 240-243.

Fernández Mendoza, O., \& Gonzáles Moreno, Á. (2012). Amputación, desarticulación: definición, indicaciones; niveles de amputación en miembro superior e inferior: tipos de ortesis o prótesis; amputación en niños y en enfermedades vasculares periféricas; técnicas operatorias. In O. Fernández Mendoza, \& Á. Gonzáles Moreno, Cirugía Radical en el Aparato Locomotor .

Gabor, D. (1946). Theory of communication. J Inst Elect Eng, 429-457.

García Ruiz, A. S., \& Fernández Moreno, A. (2005). La inclusión para las personas con discapacidad: entre la igualdad y la diferencia. Ciencias de la salud.

González, D. (2004). Robótica y Prótesis Inteligentes. Revista Digital Universitaria.

Guglielminotti, P., \& Merletti, R. (1992). Effect of electrode location on surface myoelectric signal variables: a simulation study. 9th Int. Congress of ISEK.

Highsmith, M. J., Highsmisth, J. T., \& Kahle, J. T. (2011). Identifying and Managing Skin Issues With Lower-Limb Prosthetic Use. inMOTION.

Hiraiwa, A., Shimohara, K., \& Tokunaga, Y. (1989). EMG Pattern Analysis and Classification by Neural Network. Conference Proceedings., IEEE International Conference on Systems, Man and Cybernetics, 1113-1115.

Holzer, L. A., Sevelda, F., Fraberger, G., Bluder, O., Kickinger, W., \& Holzer, G. (2014). Body Image and SelfEsteem in Lower-Limb Amputees. PLOS One, 1-8. 
Hong-liu, Y., Pan-pan, L., \& Jia-hua, H. (2010). Development of Upper Limb Prosthesis Technology. Proceedings of the 4th International Convention on Rehabilitation Engineering \& Assistive Technology, 1-5.

House, B., Malkin, J., \& Bilmes, J. (2009). The VoiceBot: A Voice Controlled Robot Arm. Proceedings of the SIGCHI Conference on Human Factors in Computing Systems, 183-192.

Huang, H., Sun, Y., Yang, Q., Zhang, F., Zhang, X., Liu, Y., . . . Sierra, F. (2010). Integrating Neuromuscular and Cyber Systems for Neural Control of Artificial Legs. ICCPS '10 Proceedings of the 1st ACM/IEEE International Conference on Cyber-Physical Systems, 129-138 .

Hunter, G., Heger, H., \& Millstein, S. (1986). Prosthetic use in adult upper limb amputees: a comparison of the body powered and electrically powered prostheses. Prosthetics and Orthotics International, 27-34.

INEI. (2012). Primera Encuesta Nacional Especializada sobre Discapacidad. Lima: Instituto Nacional de Estadística e Informática.

Ñique, V. A. (2011). Análisis sobre la difusión de la Robótica Industrial en Latinoamérica. Revista Iberoamericana de Automática e Informática industrial, 1-4.

Ismail, A. R., \& Asfour, S. S. (1998). Continuous wavelet transform application to EMG signals during human gait. Thirty-Second Asilomar Conference on Signals, Systems \& Computers, 325-329.

Khotanzad, A., \& Hua Hong, Y. (1990). Invariant Image Recognition by Zernike Moments. IEEE Transactions on Pattern Analysis and Machine Intelligence, 489 - 497.

Khushaba, R. N., Kodagoda, S., Takruri, M., \& Dissanayake, G. (2012). Toward improved control of prosthetic fingers using surface electromyogram (EMG) signals. Expert Systems with Applications, 10731-10738.

Krogh, A., \& Vedelsby, J. (1994). Neural Network Ensembles, Cross Validation, and Active Learning. NIPS'94 Proceedings of the 7th International Conference on Neural Information Processing Systems, 231-238.

Krogh, A., \& Vedelsby, J. (1994). Neural Network Ensembles, Cross Validation, and Active Learning. NIPS'94 Proceedings of the 7th International Conference on Neural Information Processing Systems, 231-238.

Kumar, D. K., Pah, N. D., \& Bradley, A. (2003). Wavelet analysis of surface electromyography to determine muscle fatigue. IEEE Trans Neural Syst Rehabil Eng, 400-406.

Laterza, F., \& Olmo, G. (1997). Analysis of EMG signals by means of the matched wavelet transform. Electronics Letters, 357-359.

Li, S. Z., \& Lu, J. (1999). Face Recognition Using the Nearest Feature Line Method. IEEE TRANSACTIONS ON NEURAL NETWORKS, 439-443.

Light, C. M., Chappell, P. H., Hudgins, B., \& Engelhart, K. (2002). Intelligent multifunction myoelectric control of hand prostheses. Journal of Medical Engineering \& Technology, 139-136.

López, N. M., di Sciascio, F., \& Soria, C. M. (2009). Robust EMG sensing system based on data fusion for myoelectric control of a robotic arm. Biomed Eng Online.

Lyman, J. (1974). Design Approaches to Adaptive Control Systems for Arm Prostheses. ACM '74 Proceedings of the 1974 annual ACM conference - Volume 2, 515-519.

Marcel Dekker, Inc. (2002). Image Recognition and Classification Algorithms, Systems, and Applications. New York: Marcel Dekker, Inc.

Mazgaj, M. (2016). Limb amputation. Nursing Standard, 61.

Mitchell, T. M. (1997). Machine Learning. New York: McGraw-Hill Science/Engineering/Math.

Nakano, K. (1997). Application of neural networks to the color grading of apples. Computers and Electronics in Agriculture, 105-116.

Orabona, F., Castellini, C., Caputo, B., Fiorilla, A. E., \& Sandini, G. (2009). Model Adaptation with Least-Squares SVM for Adaptive Hand Prosthetics. IEEE International Conference on Robotics and Automation.

Pang-Ning, T., Steinbach, M., Karpatne, A., \& Kumar, V. (2005). Cluster Analysis: Basic Concepts and Algorithms. In T. Pang-Ning, M. Steinbach, A. Karpatne, \& V. Kumar, Introduction to Data Mining (pp. 487-528). Noida: Pearson.

Pattichis, C. S., \& Pattichis, M. S. (1999). Time-scale analysis of motor unit action potentials. IEEE Trans Biomed Eng., 1320-1329. 
Raez, M. B., Hussain, M. S., \& Mohd-Yasin, F. (2006). Techniques of EMG signal analysis: detection, processing, classification and applications. Biological Procedures Online, 11-35.

Reddy, C. K., \& Vinzamuri, B. (2013). A Survey of Partitional and Hierarchical Clustering Algorithms. In C. C. Aggarwal, \& C. K. Reddy, Data Clustering: Algorithms and Applications (pp. 87-110). Boca Raton: CRC Press.

Rosenblatt. (1959). Principles of Neurodynamics. Spartan Books.

Ruck, D. W. (1990). Characterization of Multilayer Perceptrons and their Application to Multisensor Automatic Target Detection. AIR FORCE INSTITUTE OF TECHNOLOGY, 3-119.

Salas Casapino, C. A. (2014). Diseño de una Prótesis Mioeléctrica para desarticulación de muñeca. Lima: Pontificia Universidad Católica del Perú.

Schalk, G., \& Leuthardt, E. C. (2011). Brain-Computer Interfaces Using Electrocorticographic Signals. IEEE Reviews in Biomedical Engineering, 140-154.

Scheme, E., Fougner, A., Stavdahl, O., Chan, A. C., \& Englehart, K. (2010). Examining the adverse effect of limb position on pattern recognition based myoelectric control. Proceedings of the 32nd annual international conference of the IEEE EMBS, 6337-6340.

Schoppen, T. (2002). Functional outcome after a lower limb amputation. RIJKSUNIVERSITEIT GRONINGEN.

Shalev-Shwartz, S., \& Ben-David, S. (2014). Understanding Machine Learning: From Theory to Algorithms. New York: Cambridge University Press.

Shenoy, P., Miller, K. J., Crawford, B., \& Rao, R. P. (2008). Online Electromyographic Control of a Robotic Prosthesis. IEEE TRANSACTIONS ON BIOMEDICAL ENGINEERING, 1128-1135.

Simonyan, K., \& Zisserman, A. (2015). Very Deep Convolutional Networks for Large-Scale Image Recognition. ICLR 2015.

Sirovitch, L., \& Kirby, M. (1987). A Low-DimensionalProcedurefortheCharacterizationofHu- man Faces. Journ. of Optical Society of America, 519-524.

Soria, C., Freire, E., \& Carelli, R. (2006). Stable AGV corridor navigation based on data and control signal fusion. Latin American Applied Research, 71-78.

Tae-Kyun, K., Kittler, J., \& Cipolla, R. (2007). Discriminative Learning and Recognition of Image Set Classes Using Canonical Correlations. IEEE TRANSACTIONS ON PATTERN ANALYSIS AND MACHINE INTELLIGENCE, 1005-1018.

Tariq, M., Koreshi, Z., \& Trivailo, P. (2017). Optimal Control of an Active Prosthetic Ankle. ICMRE 2017 Proceedings of the 3rd International Conference on Mechatronics and Robotics Engineering, 113-118.

TensorFlow. (2018, 06 07). TensorFlow. Retrieved from TengorFlow: https://www.tensorflow.org/

Thoma, M. (2017). Analysis and Optimization of Convolutional Neural Network Architectures. Karlsruhe: Department of Computer Science Institute for Anthropomatics and FZI Research Center for Information Technology.

Timmermans, A. J., \& Hulzebosch, A. A. (1996). Computer vision system for on-line sorting of pot plants using an artificial neural network classifier. Computers and Electronics in Agriculture, 41-55.

Torrey, L., \& Shavlik, J. (2009). Transfer Learning. Handbook of Research on Machine Learning Applications.

Van Gerven, M. (2017). Computational Foundations of Natural Intelligence. Frontiers in Computational Neuroscience, 7-30.

Wang, D., Dowell, F. E., \& Lacey, R. E. (1999). Single wheat kernel color classification using neural networks. Transactions of the ASAE, 233-240.

Winter, D. (1984). Pathologic gait diagnosis with computer-averaged electromyographic profiles. Arch Phys Med Rehab, 393-398.

Wright, D. R. (2005). Finite State Machines. CSC215 Class Notes, 1-28.

Yamada, R., Ushiba, J., Tomita, Y., \& Masakado, Y. (2003). Decomposition of Electromyographic Signal by Principal Component Analysis of Wavelet Coefficient. IEEE EMBS Asian-Pacific Conference on Biomedical Engineering 2003, 118-119. 
Yanagisawa, T., Hirata, M., Saitoh, Y., Goto, T., Kishima, H., Fukuma, R., . . Yoshimine, T. (2011). Real-time control of a prosthetic hand using human electrocorticography signals. Journal of Neurosurgery, 1715-1722.

Yang, C. C., Prasher, S. O., \& Mehuys, G. R. (1997). An artificial neural network to estimate soil temperature. Canadian Journal of Soil Science, 421-429.

Yang, C. C., Prasher, S. O., Landry, J. A., Ramaswamy, H. S., \& Ditommaso, A. (2000). Application of artificial neural networks in image recognition and classification of crop and weeds. Canadian Biosystems Engineering, 147-152.

Zennaro, D., Welling, P., \& Koch, V. M. (2003). A Software Package for the Decomposition of Long-Term Multichannel EMG Signal Using Wavelet Coefficients. IEEE Trans Biomed Eng. 2003, 58-69.

Zhang, X., Huang, H., \& Yang, Q. (2012). Implementing an FPGA system for real-time intent recognition for prosthetic legs. DAC '12 Proceedings of the 49th Annual Design Automation Conference, 169-175. 